\title{
Fractal and Chaos Characteristics of Acoustic Emission During Failure of Cemented Filling Body
}

\author{
B.W. $\mathrm{Hu}^{1,2^{*}}$, C.H. $\mathrm{Li}^{1,2}$ and Z.C. $\mathrm{Li}^{3}$ \\ ${ }^{1}$ Key Laboratory of the Ministry of Education of China for High-efficient Mining and Safety of Metal Mines, University of Science and \\ Technology Beijing, Beijing 100083, China \\ ${ }^{2}$ School of Civil and Environmental Engineering, University of Science and Technology Beijing, Beijing 100083, China \\ ${ }^{3}$ School of Mining Engineering, University of New South Wales, Sydney, 2052, Australia
}

Received 27 March 2014; Accepted 2 September 2014

\begin{abstract}
The cemented filling body is taken as the object of this study, whose nonlinear dynamic characteristics of damage and instability are researched by virtue of acoustic emission (AE) under uniaxial cyclic loading and unloading test. The results show that there exists similarity in variation trend between correlation dimension and maximum Lyapunov exponent during each loading stage, which also verifies the disorder and chaos often reflect the complexity of fractal. Before the filling body gets to the instability, the correlation dimension declines continuously. So the filling body's unstable failure is a kind of ordered dimension reduction process. Such kind of characteristic can be as a criterion condition for filling body's failure. The maximum Lyapunov exponent varies little in magnitude, therefore, it can't be apt to be used as the criterion condition, but it can be used to predict the AE time series that is of chaos feature, and through it higher forecast precision can be got.
\end{abstract}

Keywords: Cemented Filling Body, Nonlinear Dynamics, Fractal, Lyapunov Exponent, Acoustic Emission

\section{Introduction}

A failure process of rocks and concrete material is usually characterized by crack initiation, propagation, and coalescence with associated damage and evolution. In this process, the Acoustic emission (AE) as a stress wave is often generated. As to the analysis methods of AE signal, the characteristic parameters such as the frequency of AE events, amplitude, energy ratio, etc. were often main points that researchers cared about in the beginning and then the deformation and failure mechanisms of materials were studied by analyzing the variation law of those parameters [1]. For instance, there would take on AE quite period before the failure of the rock materials. However, the field monitoring and laboratory test results showed that the appearance of the quite period would not always mean the failure, and how to confirm the critical value of parameters as a precursor of failure also was a difficult and key question $[2,3]$.

After that, many researchers turned to nonlinear science field in which the failure and damage mechanisms of rock and concrete material were analyzed and discussed [4]. L. Kortas collected many sets of AE data referring to seismic phenomena of different origin [5]. These sets collected were related to fracturing: starting from a microscale, i.e., seismoacoustic emission registered in rock samples subjected to compression, through mining induced seismicity, to a macroscale that was represented by earthquakes. The data were examined in terms of the presence of non-linear

\footnotetext{
*E-mail address: hu_bao_wen@aliyun.com

ISSN: 1791-2377 (C) 2014 Kavala Institute of Technology. All rights reserved.
}

dynamics and deterministic chaos, and the results showed it was applicable for description of seismological processes and evaluation of seismological hazard by adopting nonlinear theory. A. Carpinteri et al. found a simple relation between $b$ and fractal dimension of the crack network in concrete $[6,7]$. As a result, the b-value analysis in $\mathrm{AE}$ monitoring tests permitted evaluation of dimension of the damaged domain. Subsequently, by virtue of three-point bending and uniaxial loading tests, they found fractal dimension would decrease from an initial value comprised between 2 and 3 to a final value nearly equal to 2 during the damage process, but a single fractal dimension didn't adequately describe a crack network, since two damaged domains with the same fractal dimension could have significantly different properties. J.D. Hogan et al. studied on dynamic fragmentation of granite for impact energy of 6$28 \mathrm{~J}$, which confirmed the fractal value of the cumulative distribution of fragment sizes indicated that the comminution was a domain fragmentation mechanism [8]. H.P. Xie et al. analyzed the fractal property of spatial theory of acoustic emission during the bedded rock salt damage and failure process by utilizing the column covering method, and established the relationships of stress and energy release with the fractal dimension [9]. Therefore it was possible to forecast the failure of rock by variation of the relationships. R.F. Yuan et al. also calculated fractal dimension of AE spatial distribution by using box-counting method, and thought the fractal value could be as a criterion for rock failure [10]. X.P. Zhou et al. calculated the correlation dimension and the Largest Lyapunov exponents of AE, and thought the failure process of rock possessed the chaotic 
characteristics and the correlation dimension could be as a failure evaluation [11]. There were other researches on fractal of rock damage that demonstrated the fractal value was important for forecasting rock failure and understanding the mechanism of damage $[12,13]$.

The researches on fractal and chaos mentioned above belong to the scope of nonlinear dynamics which involves a wide range of engineering application. However, in current studies, the chaos and fractal characteristics are usually separately studied, and not analyzed contrastively. The variation law of largest Lyapunov exponent with the evolution of damage of materials is also not studied. Therefore, based on the previous studies, we chose the cemented filling body as the research object to investigate its nonlinear dynamic characteristics existing in the process of damage evolution by means of acoustic emission under uniaxial compression test. It will be meaningful for filling mining methods in both theory and engineering application.

\section{Design of Experiment and Acquisition of Data}

The aggregates of cemented filling body were fetched from classified tailings of a certain mine. The grain size is 74.5 um. Take P.O. 42.5 (according to china standard GB1751999) silicate cement as the cementing materials. The cement-sand ratio, mass concentration, and size of the cylindrical specimens are $1: 4,71 \%$, and $50 \mathrm{~mm} \times 100 \mathrm{~mm}$ respectively. 16 specimens were manufactured, of which 7 ones were used in this experiment, the rest for other research contents (Fig. 1).

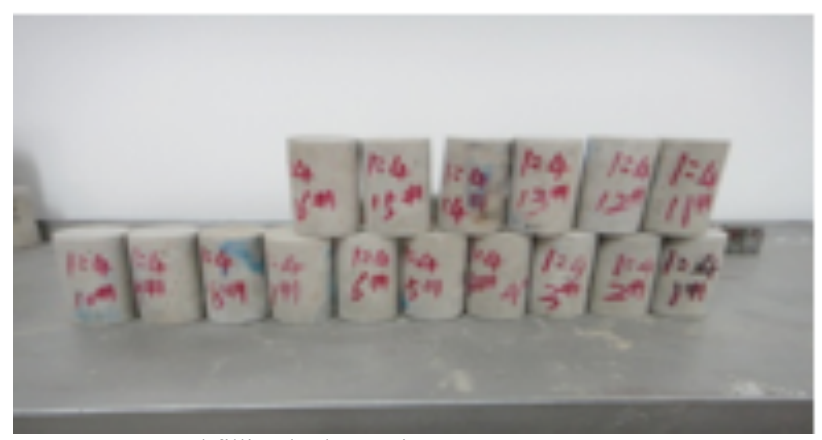

Fig.1. Cemented filling body specimens

The uniaxial cyclic loading and unloading test was performed in stress control by servo-controlled hydraulic test machine with maximum capacity of $1000 \mathrm{kN}$ (Model: RMT$105 \mathrm{C})$. In this work the loading and unloading rate was set $0.01 \mathrm{kN} / \mathrm{s}$. The whole loading process was divided into four cycle loading and unloading stages in which the lower stress limit was kept invariant and the upper stress limit was gradually increased. The first three upper loading stress limits were kept $40 \%, 60 \%$, and $80 \%$ of strength of the cemented filling body respectively. The specimens were loaded up to failure in the fourth loading stage.

Fig. 2 shows the test system, the left hand side is servocontrolled hydraulic test machine. The upper right corner is the loading location magnified, as we can see the white apparatus fixed in the specimen is $\mathrm{AE}$ transducer. The bottom-right is AE monitoring system (Model: SAEU2SAE) used for filtering noise and real time acquiring the $\mathrm{AE}$ signals from the transducers. The monitoring system manufactured by Beijing Soundwel Technology Ltd. is composed of AE transducer, pre-amplifier, signal acquisition, processing, recording, and display, which can real time acquire and display $\mathrm{AE}$ signals and characteristic parameters. The sampling length, sampling interval and preamplifier gain were set to 2048 Byte, $60 \mu$ s and $40 \mathrm{~dB}$ respectively in this test. The $\mathrm{AE}$ sensor with frequency sensitivity between $60 \mathrm{kHz}$ to $400 \mathrm{kHz}$ and a central frequency of $150 \mathrm{kHz}$ was used in this experiment. Finally the data of 6 specimens were obtained, the others failed.

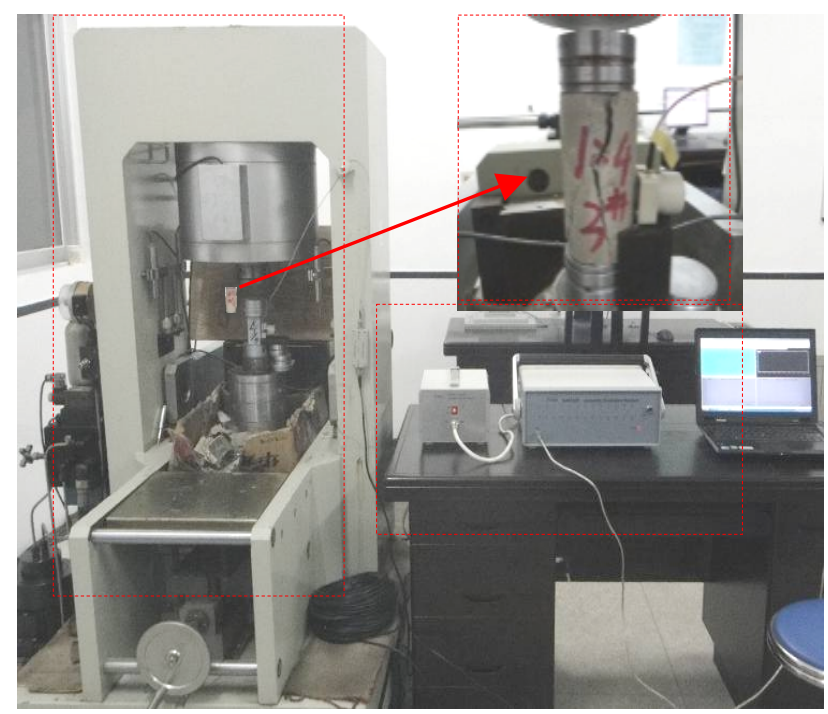

Fig.2. Experiment device

\section{Fractal and Chaos Analysis of Acoustic Emission}

From the previous studies we know, the damage processes of rock and concrete are characterized by the progressive coalescence of microcracks to form fracture surfaces, furthermore, these microcraks are constituted of more submicrocraks [2]. Such kinds of self-similar behaviors will lead to fragmental distribution and energy dissipation having self-similarities which are known as the fractal characteristics. Therefore, the fractal dimension of AE can be used as measurement of disorder and irregularity of microcracks inside rock, and also reflects the evolution law of microcracks. P. Grassberger et al. suggested an easy way of calculating the correlation dimension to describe the selfsimilarity of nonlinear dynamic system, which is widely adopted by many researchers in current [14].

A single variable time series of deterministic nonlinear system often contains the chaos behaviors. The largest Lyapunov exponent is the measure of trajectory divergence during system evolution, which reflects the global chaos level. If the largest Lyapunov exponent is positive, then the dynamic system is chaotic. This is one way to determine whether a system is chaotic or not. In this paper, we investigate the evolution law of the largest Lyanpunov exponent and fractal dimension with time during the whole damage process of the cemented filling body, and the correlative relationships between them.

\subsection{Algorithmic Method of Correlation Dimension}

The correlation dimension of AE amplitude is calculated by G-P method. The detail calculation steps are as follow:

1) Let set of observations of scalar time series be $\{x(t), t=1,2, \ldots N\}$. Then the phase space reconstruction from the time series can be implemented according to Tokens theorem: 
$X_{i}(t)=\left\{x_{i}(t), x_{i}(t+\tau), \ldots, x_{i}[t+(m-1) \tau]\right\}$

$(i=1,2, \ldots, M)$

Where $\tau$ is time delay, $m$ is the embedding dimension, $X_{i}(t)$ is a phase point in the phase space, and $M=N-(m-1) \tau$ is the number of phase points in the phase space. The $\tau$ is calculated by mutual information [16].

2) The correlation sum $C_{n}(r)$ for a set of $M$ points in phase space is given by this formula:

$$
C_{n}(r)=\frac{1}{M^{2}} \sum_{i, j=1}^{M} \theta\left(r-\left\|X_{i}-X_{j}\right\|\right)
$$

Where $\theta$ is the Heaviside function. The double sum counts the number of pairs of points whose distance apart is less than $r$, divided by the total number of pairs which is possible to form with the $M$ points. As $r \rightarrow 0$, there exists a formula supported, $\lim C_{n}(r) \propto r^{D}$. The $D$ is the correlation dimension.

3) In the practical application, the different $m$ value should be chosen for reconstructing phase space so as to observe the variation of $D$ with $m$. As the $D$ converges to a certain value, it can be as the final correlation dimension and $m$ as the final embedding dimension. In this paper, each correlation dimension is calculated by 600 consecutive AE amplitude data.

\subsection{Algorithmic Method of Maximum Lyanpunov Exponent}

The largest Lyanpunov exponent is calculated by small-data method that is put forward by Rosenstein [15]. The detail calculation steps are as follow:

1) Estimate the mean period of the original time series using FFT.

2) Reconstruct the phase space from time series of $\mathrm{AE}$ amplitude, according to the embedding dimension $m$ and time delay $\tau$ that has been determined above.

3) Locate the nearest neighbor $X_{\hat{i}}$ of each phase point, $X_{\hat{i}}$ is in the phase space and constrain the temporal separation. This is expressed as

$$
d_{i}(0)=\min \left\|X_{i}-X_{\hat{i}}\right\|,|i-\hat{i}|>P
$$

4) Calculate the separation distance of the neighbors after the $j$ th discrete-time step for each $X_{i}$ :

$$
\begin{aligned}
& d_{i}(j)=\left|X_{i+j}-X_{\hat{i}+j}\right| \\
& j=1,2, \ldots, \min (M-i, M-\hat{i})
\end{aligned}
$$

5) For each $j$, calculate the average of $\ln d_{i}(j)$ over all values of $i$

$$
x(j)=\frac{1}{q \Delta t} \sum_{i=1}^{q} \ln d_{i}(j)
$$

Where $q$ is the number of positive $d_{i}(j)$. Then the least-square method is used to fit a line with respect to Eq. 5, in which the slope of the line is the largest Lyanpunov exponent. In this paper, each largest Lyanpunov exponent is also calculated by 600 consecutive AE amplitude data.

\subsection{Evolution Law of Correlation Dimension and} Largest Lyanpunov Exponent

By reconstructing the phase space from time series of $\mathrm{AE}$ amplitude, the curves of correlation dimension and Lyanpunov exponent with time are finally obtained.

As is shown in Fig. 3a-c, the whole variation trends of correlation dimensions are similar with the Lyanpunov exponents. It is noted the data of curves are from the specimen 5 , there are other 4 specimens that show the similar variation law.

The initial part of the first loading stage often corresponds to the compaction state of microcracks and voids, and the larger fluctuations of correlation dimension can be represented as the disorder state of AE series [3, 5]. In the latter parts of the first loading stage, the compaction process of microcracks is basically finished, and the cemented filling specimens come into the local damage phase. It can be seen from Fig. 3a that the correlation dimensions decrease slowly in the whole time duration, which show that even the AE process is experiencing the dimension reduction, all the largest Lyanpunov exponents are greater than zero. That indicates the AE time series is chaotic, namely the trajectories of $\mathrm{AE}$ phase space are always in the divergence state, and the divergence quantity varies with time.

The second loading stage can be regarded as the evolution and growth of the microcracks. The correlation dimensions and largest Lyanpunov exponents oscillate intensively with time, which show the different scale damages inside the filling body and the complexity of chaos of AE (Fig. 3b).

In the third loading stage, the correlation dimensions increase firstly and then decrease continuously, which show the damage evolution of the filling body is coming to an unstable development in the latter part of third stage, and the specimens begin into the large scale damage (Fig. 3c). This is consistent with our experiment.

It can be said that the fourth loading stage is unstable failure stage for the filling body. The continuous decrease of the correlation dimensions confirms that the process of unstable failure is along with formation of orderly dimension reduction and dissipative structure. Therefore, such kind of variation law can be as a precursor for failure of the material. The largest Lyanpunov exponents are still greater than zero, even though the correlation dimensions are at lowest value. This means the AE process is in the chaotic state for the whole loading stage.

It is worthwhile to be noticed that there is the obvious similarity in variation trend between correlation dimensions and Lyanpunov exponents in the whole loading stage, especially in third and fourth, in spite of advance or lag between the both that appear sometimes. This confirms these seemingly highly disorder and mess features presented in the chaos dynamics reflect the complexity of fractal $[3,5]$. The largest Lyanpunov exponent is a quantity that characterizes the rate of separation of infinitesimally close trajectories in its orientation. As the exponent is less than zero, the trajectories are inclined to converge, on the contrary, separated at exponential growth rate. This explains from the mathematics view the larger positive exponent will lead to the higher complexity of geometry structure of the final trajectory in phase space, i.e., the fractal dimensions will become lager. 


\section{Prediction of Chaos Time Series for AE Amplitude}

From the results discussed above, there exists the similarity in variation trend between the both key indices, but the variation quantity of the largest Lyanpunov exponent is very small. Hence it is not appropriated to be used as the precursor for the failure of the cemented filling body in the practical application. However, as the measurement of divergence of the close trajectories, it could be a good forecasting parameter for the chaos time series in the nonlinear system $[16,17]$.

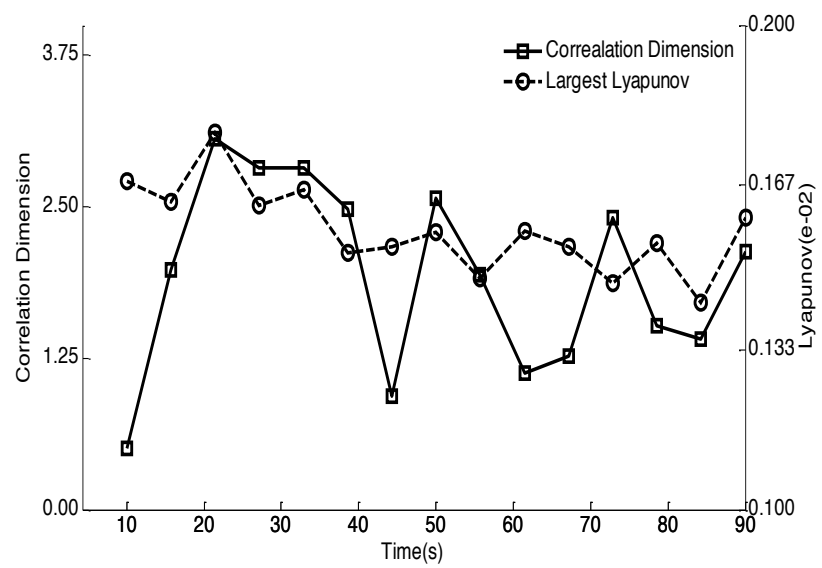

(a) First loading stage

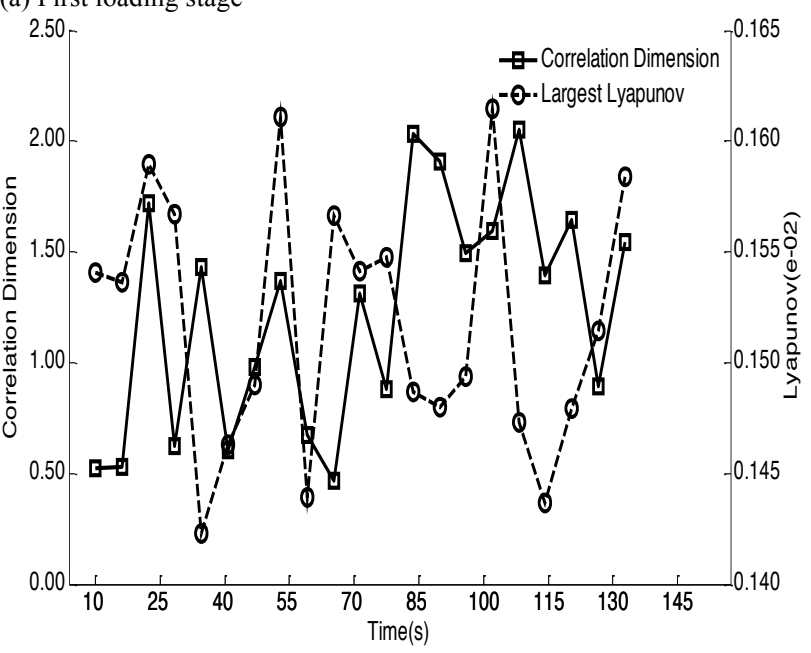

(b) Second loading stage

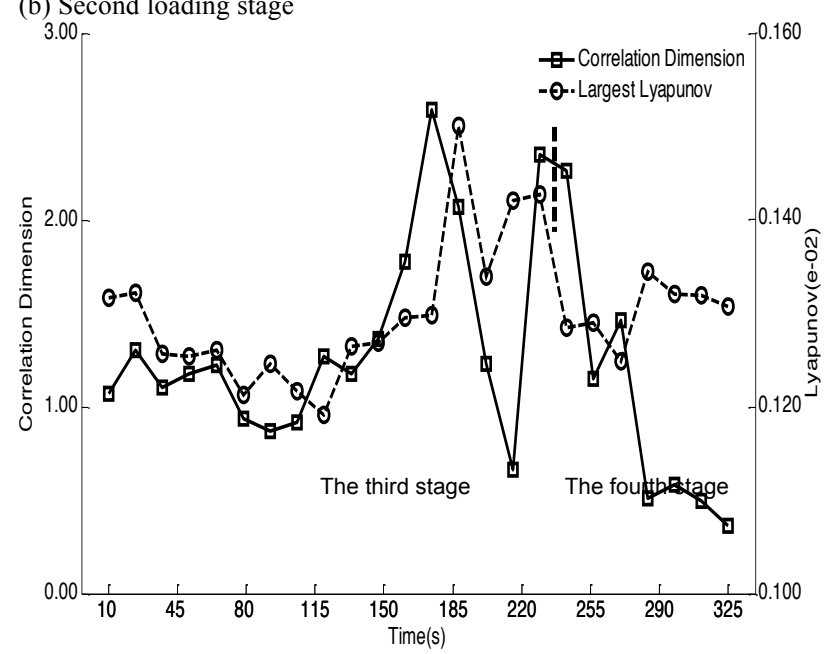

(c) Third and fourth Loading stage

Fig.3. Correlation dimension, largest Lyapunov exponent with time

\subsection{Prediction Algorithm Based on the Largest Lyanpunov Exponent}

1) Reconstruct the phase space from time series of $\mathrm{AE}$ amplitude.

2) Calculate the largest Lyanpunov exponent from the phase space.

3) Take the last phase point of phase space, $X_{M}$, as the prediction center, then search the nearest neighbor of $X_{M}$, that is $X_{K}$. Let the largest Lyanpunov exponent be $\lambda_{1}$. The following equation is given:

$\left\|X_{M}-X_{M+1}\right\|=\left\|X_{K}-X_{K+1}\right\| e^{\lambda_{1}}$

There is only one unknown component in $X_{M+1}$, i.e., $x_{M+1}[t+(m-1) \tau]$. Thus, it can be figured out by the Eq. 6 .

\subsection{Prediction Results of the AE Amplitude for the Cemented Filling Body}

In this paper, each 580 consecutive $\mathrm{AE}$ data of fourth loading stage are collected from specimen 145 and 149 respectively to predict the 20 time step results (Figs. 4a and 4c). Take the specimen 145 as an example, the time delay is 2 , the embedding dimension is 5 , the mean period is 27 , the largest Lyanpunov exponent is 0.01258 . 572 phase points are obtained by reconstructing the phase space from the 580 $\mathrm{AE}$ data, and each phase point has 5 components. The last phase point, $X_{572}=\left\{x_{572}, x_{574}, x_{576}, x_{578}, x_{580}\right\}$, is the forecasting center, and the $X_{573}=\left\{x_{573}, x_{575}, x_{577}, x_{579}, x_{581}\right\}$ is the phase point that we are prepared to predict, as we know, only $x_{581}$ is unknown. Then search the nearest neighbor of $X_{572}$ by Euclidean distance method, that is $X_{286}$. Lastly, substitute these phase points into the following equation:

$\left\|X_{572}-X_{573}\right\|=\left\|X_{286}-X_{287}\right\| e^{0.01258}$

Where $x_{581}$ can be figured out by Eq. 7. The multistep prediction can be realized through putting the $x_{581}$ back into the original AE series and repeating the above steps.

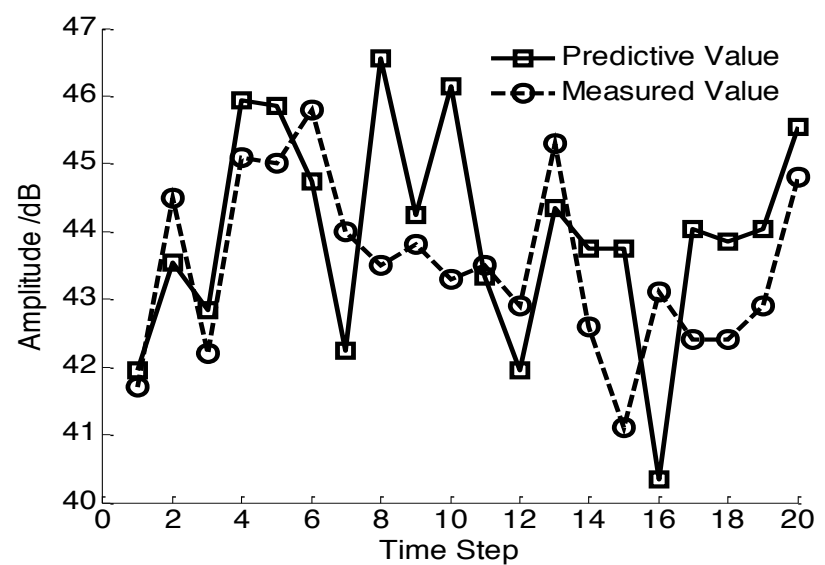

(a) The prediction results of specimen 145 


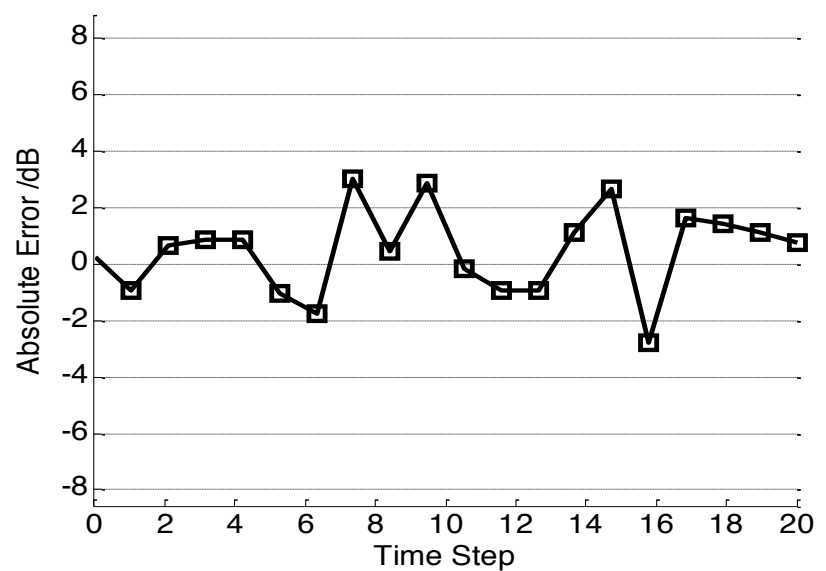

(b) The absolute error of specimen 145

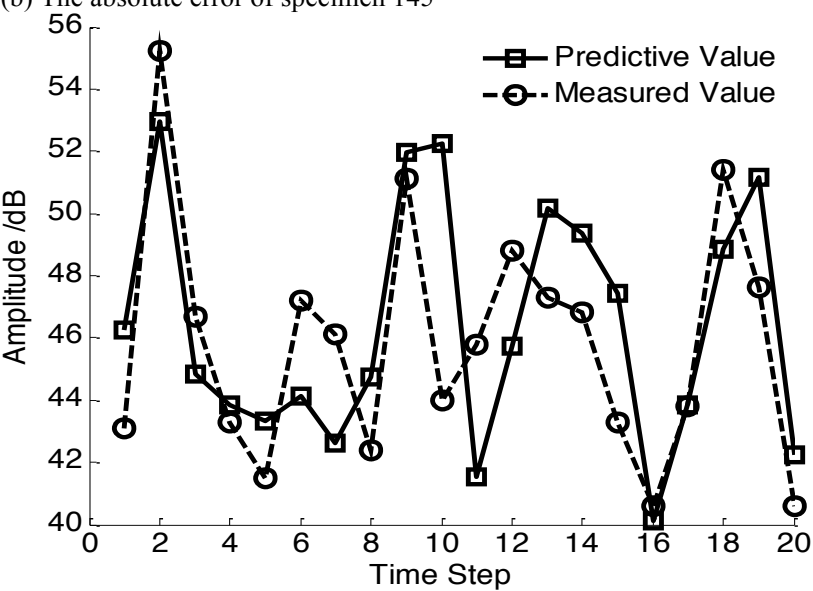

(c) The prediction results of specimen 149

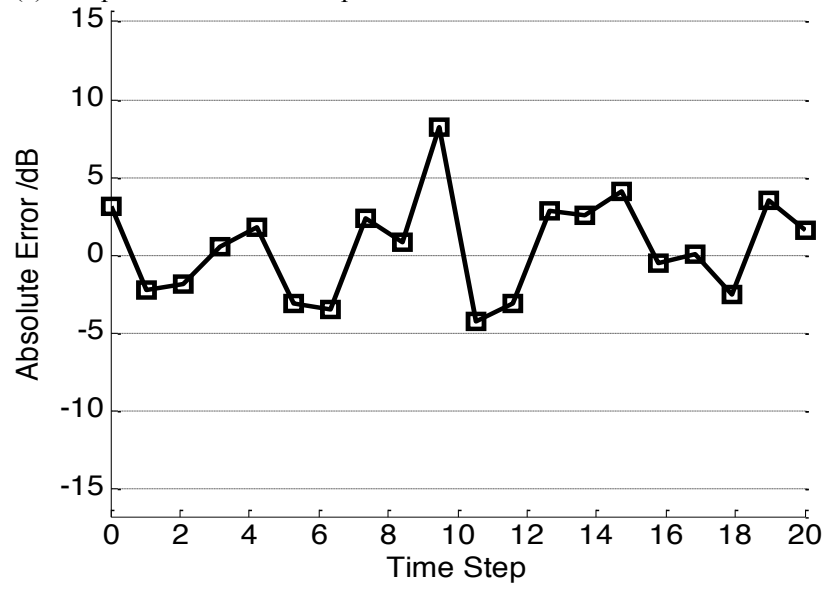

(d) The absolute error of specimen 149

Fig.4. Prediction of AE chaos time series
As shown in Fig. 4b, the absolute error of the forecast for the specimen 145 fluctuates in $-2 \mathrm{~dB}-4 \mathrm{~dB}$ range, and the specimen 149 fluctuates in $-5 \mathrm{~dB}-10 \mathrm{~dB}$ (Fig. 4d). The difference of the error is mainly caused by the largest Lyanpunov exponent. The exponent of the specimen 149 is 0.01443 , which is greater than specimen 145. Thus, the accuracy and time length of predictability for specimen 149 are less than specimen 145.

The prediction results with respect to the two specimens show that such kind of predictive accuracies can meet the requirement of engineering application. Therefore, the prediction algorithm based on the largest Lyanpunov exponent can get higher accuracy in the short term.

\section{Conclusion}

The correlation dimension and largest Lyanpunov exponent have the similarity in variation trend for each loading stage. The correlation dimension continuously decline as the cemented filling body specimens are close to the failure state, which shows the failure process of the filling body is also a dimensionality reduction and dissipative process. This law can be as the precursor for the failure.

The largest Lyanpunov exponent can't be used as the criterion for the prediction of the instability of the filling body, because its variation quantity is very small. But it can be a good forecasting parameter for the chaos time series in nonlinear system, and the chaos time series of AE can be predicted and get a higher accuracy in short term. This is meaningful for the engineering application.

\section{Acknowledgments}

This work was financially supported by National Basic Research Program of China (2010CB731501; 2010CB731506), the National Natural Science Foundation of China $(51064010,51310105020)$. All these are gratefully acknowledged.

\section{References}

[1]. Vidya S.-R. and Raghu P.-B., "A review of recent developments in parametric based acoustic emission techniques applied to concrete structures", Nondestructive Testing and Evaluation, 27 (1), 2012, pp. 47-68.

[2]. Wang S.-R., Paul H. and Cheng Y., "Experimental research on the instability characteristics of double-layer rock plates based on MTS-AE system", Applied Mathematics \& Information Sciences, 7 (1L), 2013, pp. 339-345.

[3]. Yin X.-G., Li S.-L. and Tang H., "Study on quiet period and its fractal characteristics of rock failure acoustic emission", Chinese Journal of Rock Mechanics and Engineering, $28\left(\mathrm{~S}_{2}\right), 2009$, pp. 3383-3390. (in Chinese)

[4]. Kacimi S. and Laurens S., "The correlation dimension: A robust chaotic feature for classifying acoustic emission signals generated in construction materials", Journal of Applied Physics, 106 (2), 2009, pp. 1-8.
[5]. Kortas L., "Search for chaotic dynamics manifestation in multiscale seismicity", Acta Geophysica Polonica, 53 (1), 2005, pp. 47-74.

[6]. Carpinteri A., Lacidogna G. and Niccolini G., "Morphological fractal dimension versus power-law exponent in the scaling of damaged media", International Journal of Damage Mechanics, 18 (3), 2009, pp. 259-82.

[7]. Carpinteri A., Lacidogna G. and Niccolini G., "Fractal analysis of damage detected in concrete structural elements under loading, Chaos", Solitons and Fractals, 42 (4), 2009, pp. 2047-2056.

[8]. Hogan J.-D., Rogers R.-J. and Spray J.-G., "Dynamic fragmentation of granite for impact energies of 6-28J", Engineering Fracture Mechanics, 79 (1), 2012, pp. 103-125.

[9]. Xie H.-P., Liu J.-F. and Ju Y., "Fractal property of spatial distribution of acoustic emissions during the failure process of bedded rock salt", International Journal of Rock Mechanics \& Mining Sciences, 4 (8), 2011, pp. 1344-1351. 
[10]. Yuan R.-F. and Yuan H.-L., "Fractal analysis on the spatial distribution of acoustic emission in the Failure process of rock specimens", International Journal of Minerals, Metallurgy and Materials, 16 (1), 2009, pp. 19-24.

[11]. Zhou X.-P. and Liu Q.-Y., "Research on chaotic characteristics of rock acoustic emission", Rock and Soil Mechanics, 18 (3), 2010, pp. 815-820. (in Chinese)

[12]. Yin X.-G., Li S.-L. and Tang H.-Y., "Study on Strength Fractal Features of acoustic emission in Process of Rock Failure", Chinese Journal of Rock Mechanics and Engineering, 24 (19), 2005, pp. 114-118. (in Chinese)

[13]. Ji H.-G, Wang J.-C. and Shan X.-Y., "Fractal characteristics of AE process of concrete material and application to the fracture analyses", Chinese Journal of Rock Mechanics and Engineering, 20 (6), 2001, pp. 801-804. (in Chinese)
[14]. Grassberger P. and Procaccia I., "Characterization of strange attractors”, Physical Review Letters, 50 (5), 1983, pp. 346-362.

[15]. Wang S.-R., Hagan P. and Cheng Y., "Fractal Characteristics of Sandstone Cutting Fracture Under Mechanical Shock Loading Conditions", Applied Mechanics and Materials, 226-228, 2012, pp. 1789-1794.

[16]. Rosenstein M.-T., Collins J.-J. and De L., "A practical method for calculating largest Lyapunov exponents from small data sets", Physica D, 65 (5), 1993, pp. 117-134.

[17]. Jiang J.-Q. and Li H., "Study on rock burst forecast with forecast method based on chaotic time series", Chinese Journal of Rock Mechanics and Engineering, 25 (5), 2006, pp. 889-895. (in Chinese) 